\title{
Dynamic Finite Element Analysis of an Elevated Station-Track Structure Coupled System Under Resonance
}

\author{
Kai CUI, Lei SU
}

\begin{abstract}
In this study, an elevated station and the track structure are regarded as a coupled system, and the vibration performance of the entire system is analyzed under dynamic loading. Special attention is paid to the influence of the mechanical properties of parts of the system on its vibration performance. After a brief literature review of relevant studies on urban elevated rail transit vibration performance, a three-dimensional dynamic analysis model is established considering each component and the connections of the system; furthermore, modal analysis is applied to this model. Based on the model analysis, the vibration performance under harmonic vibration loads and moving train loads are analyzed separately. During the analysis of harmonic vibration, the effects of various parameters (e.g., rail pad stiffness, mass of elastic support block) on system vibration are considered.
\end{abstract}

Keywords: dynamic; elevated station; harmonic vibration; modal analysis; track structure

\section{INTRODUCTION}

With the rapid development of urbanization and increasing traffic pressure, elevated rail transit, which provides economic, useful, high efficiency, and rapid transportation, is receiving ever more attention and development. Simultaneously, elevated stations are being generated with the development of these systems. When high-speed trains cross through densely populated areas, the vibration of the elevated station induced by the traintrack structure may cause negative structural effects.

During the past several years, many researchers have analyzed the generation and propagation of train-induced vibrations in elevated rail systems. Kaynia et al. [1] outlined a test program to measure vibrations in the ground and rail embankment induced by a high-speed train. Xia et al. [2] studied the influences of train-induced vibrations on the surrounding environment and nearby buildings through an in situ experiment. Ju et al. [3] investigated the characteristics of the ground vibration induced by moving trains on elevated railways using a number of field measurements at various train speeds. Tanabe et al. [4] analyzed the dynamic interaction of a high-speed train and railway structure in an earthquake by using the finite element method (FEM). Yang et al. [5] analyzed train induced vibrations on elevated railway station, including the vibration and characteristics vibration distrbution in the station system. Considering the pile-soil interaction, Ren et al. [6] analyzed the seismic performance of a singlecolumn elevated station by using the bilinear spring model. Tanabe et al. [7] presented an efficient computational method for analyzing the dynamic interaction of a highspeed train and railway structure, including derailment during an earthquake. Very few researchers have previously analyzed the elevated station and the track structure as a system. Because the track structure is located in the upper layer of the station under the moving train load, the station will inevitably produce vibration [8]. Therefore, the elevated station and track structure are inherently coupled, so they should be studied together as a system.

Many researchers have used FEM to study the dynamic vibrations of structures. Taktak et al. [9] presented a finite element model for the dynamic analysis of a cylindrical isotropic helical spring. The hybrid-mixed formulation was used to compute the stiffness matrix. Zhu et al. [10] developed a hybrid method combining finite element analysis and energy finite element analysis to predict the midfrequency vibrations of built-up structures and derived the associated general formulations. Chan et al. [11] found that the convergence rate of the natural frequencies for the lumped mass discretization was related to the power used to predict the free and forced vibrations of the system. Lou et al. [12] found that dynamic analysis can be used for the dynamic response of Timoshenko or Bernoulli-Euler beams that are subjected to various boundary conditions in the form of moving concentrated forces. Jorge et al. [13] performed finite element analyses to evaluate the displacement amplitudes and the critical velocities of the load on a UIC-60 rail and their dependence on the difference between the foundation's moduli in compression and in tension. Al-Khafaji et al. [14] found that structures of finite element analysis are often subjected to additional loads (e.g., air-blast loading, underwater shock) that necessitate a thorough dynamic response analysis. Wang et al. [15, 16, 17] analyzed the highfrequency dynamic behavior of plates in thermal environments, in which the thermal effects are considered to change the membrane stress state and temperature dependent material properties of the plates.

In this paper, the combined elevated station and track structure are studied using FEM. The space elevated station, regarding the elevated station and the track structure as a system is described. A three-dimensional dynamic model is then developed. The vibration performance of the total system under dynamic effects is analyzed by using the finite element method. Namely, the dependence of the vibration performance on various parameters, such as the stiffness of rail pad and the mass of elastic support block, is studied.

\section{THE ELEVATED STATION-TRACK STRUCTURE SYSTEM}

\subsection{System Description}

The structural mechanics performance of the bridgetype stations is well understood. The structure of the bridge-type station is close to the bridge, so the structural analysis of the bridge-type station is connected to that of the bridge. Therefore, those structures are not considered 
in this article. In contrast, the frame-type station structural performance is unique, and there are many applications in the actual world. This kind of structure will be the main object of study in this article. The frame-type station is different from an ordinary station, which must bear the dynamic load of railway trains, so the demands for structure vibration control are much stricter.

The dynamic response of the main station structure of the elevated station from the running control must be reduced by vibration-reducing measures. As it directly bears the dynamic load of the train, the track structure is the first layer of vibration reducing structure. Therefore, the vibration reducing characteristics of the track structure directly affect the entire station. The reasonable selection and layout of the rubber bearings that connect the track beam and the frame structure have a significant effect on the dynamic response of the lower frame structure. The arrangement, structure mass, and damping coefficient of the components also affect the vibration characteristics of the station structure.

The vibration system of the urban rail transit elevated station, which comprises the vehicle, track structure, bridge, and main structure of the rail station (main body framework, platform and station hall, etc.), is a complex system. It is considerably difficult and quite complex to accurately simulate every detail of the huge system. Therefore, it is necessary according to the purpose of the study and the premise of precision to appropriately simplify some parts of the system.

This study focuses on the vibration effect on the elevated station due to the track structure and bridge bearing parameters. The vibration is transmitted from the rail fastener and base plate to the track beam and then from the bridge bearings to the main frame structure, thereby causing the vibration of the station. Therefore, the interaction model of the elevated station and the track structure system can be simplified into the frame structure, ignoring the platform station hall, mainly considering the influence of the rail pad, bearing block under the cushion, and bridge bearings.

\subsection{System Model}

The main body frame structure of the station is selected from a three-layer elevated station in the Shanghai pearl line. The selected platform is an island platform and the transverse framework consists of three columns with two spans. The total length of the built station is $150 \mathrm{~m}$, and a settlement joint is set in the central longitudinal direction, dividing the station into two parts. Each part contains 6 cross beams, and each cross is $12 \mathrm{~m}$ long; thus, the total length is $72 \mathrm{~m}$. The width of the station is $18 \mathrm{~m}$, and the heights of the first two layers are both $5 \mathrm{~m}$. The second layer is the station hall, and the third layer is the platform.

A few elevated stations have major breakthroughs in the structure styles, such as the satellite of the third phase project of Line 4 of the Changchun ty rail transit viaduct station and Line 1 of the city Chongqing university town station structure, and can be categorized as combined or detached "bridge-building" structures, as shown in Fig. 1.

The track beams are reinforced continuous concrete box-section beams whose heights are $0.7 \mathrm{~m}$. The bottom plate width is $3.0 \mathrm{~m}$. The longitudinal direction has 6 spans, and the length of each span is $12 \mathrm{~m}$, which corr esponds with the frame structure at the bottom. According to the island platform arrangement, the buildings are on both sides. The track beam is attached to rubber bearings on the main frame cross-beam, and the loading on the track beam is transmitted to the main frame cross-beam through the rubber bearings. Fixed hinge bearings are used between the first and second spans and between the fifth and the sixth spans. Movable bearings are used on the other parts of the station. The specific arrangement is shown in Fig. 2. The fixed hinge bearings can only move via vertical rotation. The movable bearings can move via vertical rotation and vertical translation.

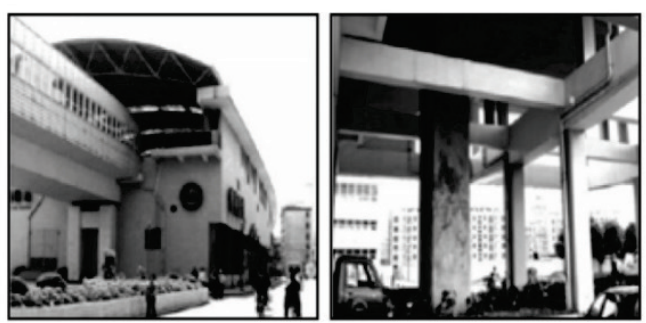

Figure 1 A combined "bridge-building" station and a detached "bridge-building" station

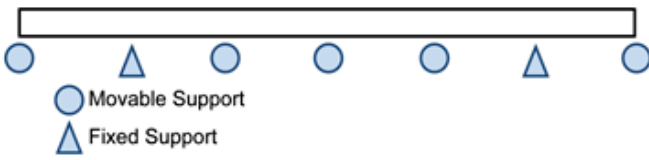

Figure 2 Arrangement of bridge bearings

The track structure has a low vibration track(LVT), which has concrete short sleepers set into a certain thickness of rubber boots. The high elastic shion layer is under the concrete short sleeper, which is poured into the ballast bed. The rubber boots play the role of isolation and the elastic cushion layer plays the role of vibration reduction. The biggest advantages of this kind of track structure are as follows. First, the rubber layer provides enough flexibility to provide the support stiffness of the steel rail below that of the ballasted track. Second, the rubber boots can also provide a certain amount of elasticity in the vertical and horizontal directions close to the ballasted track. There are a large number of applications for low vibration track structures in tunnels, and good vibration reduction effects are obtained coupled with reduced maintenance workload. In recent years, the LVT has been employed in some elevated station systems, which have with higher vibration-reduction requirements in domestic regions.

The trains are single section light-rail. The loading of the trains is arranged as shown in Fig. 3. The axle-weight of the train is $160 \mathrm{kN}$. Furthermore, the calculation diagram of the model is shown in Fig. 4.

The railway model mainly includes the beam model and the finite element model. At present, the continuous elasticity railway beam model also includes two forms: the Euler beam model and the Timoshenko beam model. The Euler beam model only considers rail bending deformation but not the shear deformation. The Timoshenko beam model introduces the rotary inertia of beam, considering the shear strain of the rail, which can be compared with the measured parameters, so that the calculation of the beam 
stress becomes complete. According to the data analysis, there are certain differences in the low-frequency range of these two kinds of beams. Therefore, the Timoshenko beam model is selected in this study. The finite element model is divided into a plate model, a beam-plate-beam model, and the entity model. The plate model divides the rail head, rail waist, and the rail bottom into plate units. The beam-platebeam model divides the rail head and rail bottom into beam units and divides the rail waist into a plate unit. The entity model divides the whole railway into entity units. The response of the moving load on the track structure is not considered. Although the entity model is accurate, it is too computationally intensive. The Timoshenko beam model fully reflects the basic characteristic of the rail. Therefore, the Beam-188 unit is used for the Timoshenko beam model. The form of the used cross section is a standard $60 \mathrm{~kg} / \mathrm{m}$ rail section; through Auto $\mathrm{CAD}$ drawing, the cross section can be imported by Auto CAD and ANSYS.

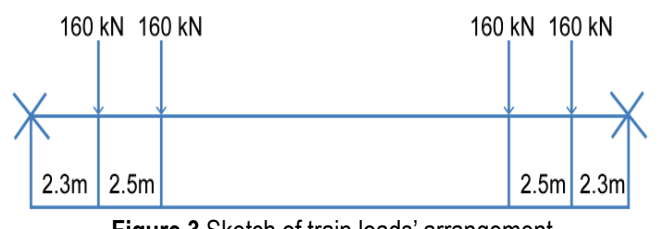

Figure 3 Sketch of train loads' arrangement

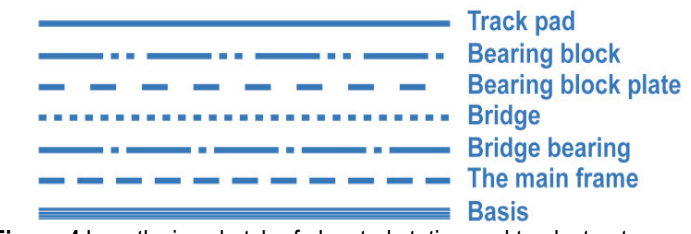

Figure 4 Lengthwise sketch of elevated station and track structure model

The rail fastener, rubber boots, and bridge bearings form the connecting parts of system, which play the roles of elastic support and vibration reduction. The reasonable selection of parameters is particularly important to the elastic and vibration reduction for the whole system. Therefore, parameter selection is the key aspect of this study. In this model, the spring damping unit Combinl4 is used in the the model. The rail fastener is discretely distributed every $0.6 \mathrm{~m}$ in the longitudinal direction. The influence of the stiffness of the rail fastener is only considered in the vertical direction. The angles of the vertical and horizontal direction and the longitudinal rotation and the vertical rotation of each node of the rail way are all set to zero. Considering the three axes of the rubber boots, the elastic damping unit can be used for its simulation. The vertical motion of the bridge bearings is simulated with the spring damping unit and the other degrees-of-freedom are coupled with the corresponding nodes of the bridge and the bearing framework.

The LVT is simulated with the mass unite Mass21, which has point elements with six degrees-of-freedom where each can have a different mass and inertia. The supporting blocks are connected with the rail and the rail beam elements by the spring damping units.

The track beam adopts the box girder structure which is widely used in urban rail transit. The advantages of this structure are that the building height is moderate and it requires less work; simultaneously, the applicability and stress on the whole building is better and the structure deforms less as it ages. The track beam in the model is simulated with entity elements Solid45, which can better reflect the characteristics of the box girder itself. Considering the influence of the ballast bed, the track bed will be placed on the bridge deck and as a part of the bridge for actual modeling.

The cross sections of the center columns, side columns, the cross beam and the longitudinal beam in the main frame structure of the station are all rectangular. Therefore, the model uses the three-dimensional beam unit Beam4, which is a uniaxial loading unit that can incorporate tension, pressure, bending. This unit accurately represents the frame structure mechanical characteristics. In the model, the structure between the columns is steel and the boundary conditions of the column bottoms are fixed so they do not consider the influence of the interaction between soil and structure.

\subsection{Modal Analysis of the Model}

Modal analysis is used to determine the natural frequencies and the natural modes under the conditions of non-damping and free vibration. The natural mode is the deformation forms of the structure corresponding to a natural frequency. The natural frequencies and modes are determined by the geometric shape, material properties, and constraints; they do no not depend on external loading conditions.

Modal analysis is a modern method for studying the characteristics of dynamic structure systems that is an application of the system identification method in the field of vibration engineering. The mode is the natural vibration characteristics of the mechanical structure and each mode has a specific natural frequency, damping ratio, and modal vibration mode. The modal parameters can be obtained by calculation or test analysis, which is called modal analysis. If the analysis process is calculated using the finite element method, it is known as computational modal analysis. If the modal parameters are obtained via system input and output signals after parameter identification, it is known as experimental modal analysis.

The vibration mode represents the inherent characteristics of the entire elastic structure. If the main modal characteristics in the frequency range of a structure can be understood by the modal analysis method, the actual frequency response of the structure under external or internal functions of the frequency can be predicted. Therefore, modal analysis is an important method for dynamic design and equipment fault diagnosis; furthermore, it is the startting for harmonic response analysis, transient dynamics analysis, spectrum analysis, and other dynamics analysis techniques.

The applications of modal analysis technology can be summarized as follows:

- evaluation of the dynamic characteristics of existing structures,

- $\quad$ prediction and optimization of the structural dynamic characteristics of new structures,

- $\quad$ prediction and diagnosis of faults in the stru- ctural system,

- control of the noise produced by a structure,

- identification of structure load.

In structure design, the primary goal of studying elastic 
body vibration is avoiding resonance. The structure can be seen as a vibrating system of multiple degrees-of-freedom with multiple natural frequencies modes. The vibration shape that corresponds to a natural frequency is the main vibration mode. The natural frequencies and main mode are only associated with the stiffness and mass distribution of structure. The mathematical model of dynamic problems can be expressed by the following system of second order simultaneous Differential equations:

$$
\boldsymbol{M} \ddot{\boldsymbol{x}}(t)+\boldsymbol{C} \dot{\boldsymbol{x}}(t)+\boldsymbol{K} \boldsymbol{x}(t)=\boldsymbol{f}(t)
$$

where $\boldsymbol{M}, \boldsymbol{C}$, and $\boldsymbol{K}$ are the mass of the system, damping, and stiffness matrices, respectively; furthermore, $\boldsymbol{M}$ and $\boldsymbol{K}$ are usually symmetric matrices, whereas $\boldsymbol{C}$ is an asymmetric matrix. The displacement vector of every point in the system is $\boldsymbol{x}(t)$, and the exciting force vector of the system is $f(t)$.

The natural frequencies and main modes are only associated with the stiffness and mass distribution of structure. Therefore, the analysis can be performed on the differential equation system without damping or an exciting force:

$$
\boldsymbol{M} \ddot{\boldsymbol{x}}(t)+\boldsymbol{K} \boldsymbol{x}(t)=0
$$

The simple harmonic vibration of the structure is as follows:

$$
\boldsymbol{x}(t)=a \sin (\varpi t+\tau)
$$

where the column vector $a=\left(a_{1}, a_{2}, \ldots, a_{n}\right)^{\mathrm{T}}$ expresses the amplitudes of the nodes, $n$ is the total degrees-offreedom. The vector is the form of the structure vibration. The time function $\sin (\varpi t+\tau)$ expresses the rule of the structure vibration on each node with the time.

Differentiating (3) twice with respect to time yields:

$$
\ddot{\boldsymbol{x}}(t)=-\varpi^{2} a \sin (\varpi t+\tau)
$$

Inserting (3)and (4) into (2) yields:

$$
\boldsymbol{K} a-\varpi^{2} \boldsymbol{M} a=0
$$

This is a generalized eigenvalue problem. When the structure is in free vibration, it must have a non-zero solution:

$$
\left|\boldsymbol{K}-\varpi^{2} \boldsymbol{M}\right|=0
$$

It is the free vibration frequency equation of the system, which is a size $\mathrm{n}$ algebraic equation about $\varpi^{2}$. Therefore, there are $\mathrm{n}$ roots of the equation which are $\varpi_{1}^{2}, \varpi_{2}^{2}, \ldots, \varpi_{n}^{2}$. There are linearly independent solutions in $\boldsymbol{K} a-\varpi^{2} \boldsymbol{M} a=0, a_{i}(i=1,2, \ldots, n)$, for each root. In the vibration analysis, $\varpi_{i}^{2}$ and $a_{i}$ are the $i^{\text {th }}$ order natural frequency of the structure and the corresponding main vibration mode, respectively.

\section{DYNAMIC ANALYSIS OF THE RESONANCE EFFECT FOR THE ELEVATED STATION-TRACK STRUCTURE SYSTEM}

\subsection{Resonance Analysis and Determination of System Damping}

The damping of the structure is an important dynamic parameter for determining the structural vibration. The damping dissipates the energy of the vibration, which is beneficial to the safety of the structure. The size of the damping is directly related to the vibration strength of the structure under dynamic load. Therefore, research on the rules of structural damping is a key factor for improving the computational accuracy of the structure dynamics.

The factors that contribute to structural damping are complex, and they can be roughly divided into three categories: material damping, friction damping, and air damping. The internal material damping is formed by the internal friction among the material molecules under vibration. Friction damping, which is also named dry friction damping and coulomb damping, is formed by the structure bearings, joint friction, and other components. Air damping is formed by the friction of the structural movement from the surrounding air.

The natures of the above kinds of damping are different. The internal material damping can make the vibration decay exponentially, whereas the structural friction damping can make the amplitude decay linearly. The nature of the air medium damping is roughly similar to that of the internal material damping.

In this article, the vibration damping of the system structure cannot be calculated as accurately as the calculation of the natural frequency. Considering the actual situation and convenience in the calculation, usually Rayleigh damping is used in structural dynamics computations. Rayleigh damping, which is also known as proportional damping, the most commonly used form of damping, and it is also relatively simple damping. Rayleigh damping is the first choice for most practical dynamics analyses, and it is often applied for many practical engineering problems.

The assumption of the Rayleigh damping is that the damping matrix $\boldsymbol{C}$ is a linear combination of the mass matrix $\boldsymbol{M}$ and the stiffness matrix $\boldsymbol{K}$ :

$\boldsymbol{C}=\alpha \boldsymbol{M}+\beta \boldsymbol{K}$

From the free vibration equation for the system, two natural frequencies are $\varpi_{i}$ and $\varpi_{j}$ and two vibration modes are $\phi_{i}$ and $\phi_{j}$. According to the orthogonality of $\phi$, the results can be expressed as follows:

$\phi_{i}^{\mathrm{T}} \boldsymbol{C} \phi_{j}=\left\{\begin{array}{cc}2 \varpi_{i} \zeta_{j}, & i=j \\ 0, & i \neq j\end{array}\right.$

The left side of the equation is multiplied by $\phi_{j}^{\mathrm{T}}$ and the the right side of the equation is multiplied by $\phi_{i}$.

The result is as follow: 


$$
\phi_{i}^{\mathrm{T}} \boldsymbol{M} \phi_{j}=\left\{\begin{array}{cc}
1, & i=j \\
0, & i \neq j
\end{array}, \quad \phi_{i}^{\mathrm{T}} \boldsymbol{K} \phi_{j}=\left\{\begin{array}{cc}
\varpi_{i}^{2}, & i=j \\
0, & i \neq j
\end{array}\right.\right.
$$

then the relationship between the undetermined coefficients, $\alpha$ and $\beta$, and the modal damping ratio can be obtained:

$$
\zeta_{k}=\frac{\alpha}{2 \varpi_{k}}+\frac{\beta \varpi_{k}}{2}
$$

where the damping ratio is $\zeta_{k}$, the natural frequency is $\varpi_{k}$ and the damping coefficients are $\alpha$ and $\beta$.

From (10), two modal damping ratios, $\zeta_{i}$ and $\zeta_{j}$, can be obtained and then the damping coefficients $\alpha, \beta$ can be obtained:

$$
\alpha=\frac{2\left(\frac{\zeta_{i}}{\varpi_{i}}-\frac{\zeta_{j}}{\varpi_{j}}\right)}{\frac{1}{\varpi_{i}}-\frac{1}{\varpi_{j}}}, \beta=\frac{2\left(\zeta_{j} \varpi_{j}-\zeta_{i} \varpi_{i}\right)}{\varpi_{j}^{2}-\varpi_{i}^{2}}
$$

When $\zeta_{i}=\zeta_{j}=\zeta$ the condition is, the damping $\alpha, \beta$ coefficients can be simplified as follows:

$\alpha=\frac{2 \varpi_{i} \varpi_{j} \zeta}{\varpi_{i}+\varpi_{j}}, \beta=\frac{2 \zeta}{\varpi_{i}+\varpi_{j}}$

Based on modal analysis, the first two frequencies can be obtained:

$\varpi_{1}=2 \pi \times 3.3079=20.7736 \mathrm{rad} / \mathrm{s}$,

$\varpi_{2}=2 \pi \times 3.8167=23.9689 \mathrm{rad} / \mathrm{s}$.

When the value $\zeta=0.05$ is taken in (12), the results are as follows:

$\alpha=1.113, \beta=0.0022$.

\subsection{Analysis of the System Resonance Effect under the Influence of the Model Parameters}

In this study, the ANSYS software is used to perform harmonic analysis of the vertical force transfer characteristics of the elevated station-track structure system. In the calculation, a load of $80 \mathrm{kN}$ (single wheel load) is applied to each rail in the central longitudinal region in the model. There is the limitation in the harmonic analysis software that all loads must have the same frequency according to the sine law of change.

The load frequency is varied from 0 to $100 \mathrm{~Hz}$. The modal analysis results show that the first frequency of the state system is $10.121 \mathrm{~Hz}$. In the computing process, a solution was found every $1 \mathrm{~Hz}$ for the 0 to $20 \mathrm{~Hz}$ range, and a solution was found every $2 \mathrm{~Hz}$ for the 20 to $100 \mathrm{~Hz}$ range. This saves computing time and computer resources while still meeting the basic requirements. The vertical force transfer characteristics of the system are analyzed by changing the parameters of the system components and extracting data for analysis of various locations: along the middle of the third and fourth crossbeams, from the top to the bottom within the rail, at the bearing block, bridges, frameworks, framework layer, and three-layer framework center pillar bottom.

\subsubsection{Effect of Cushion Stiffness Under the Rail}

The rail pad under the upper layer buffers the vertical excitation of the track through the first layer of the rail pass down vibration unit. Keeping support block mass, support block cushion stiffness, bridge support vertical stiffness, frame structure elastic modulus parameters constant, the stiffness of the rail pad is varied to analyze its influence on each part of the structure. The results of this analysis are given in Fig. 5. From the analysis, as the stiffness of the under rail pad increases, the displacement amplitudes of each part of the system decrease, but the force to the base increases.

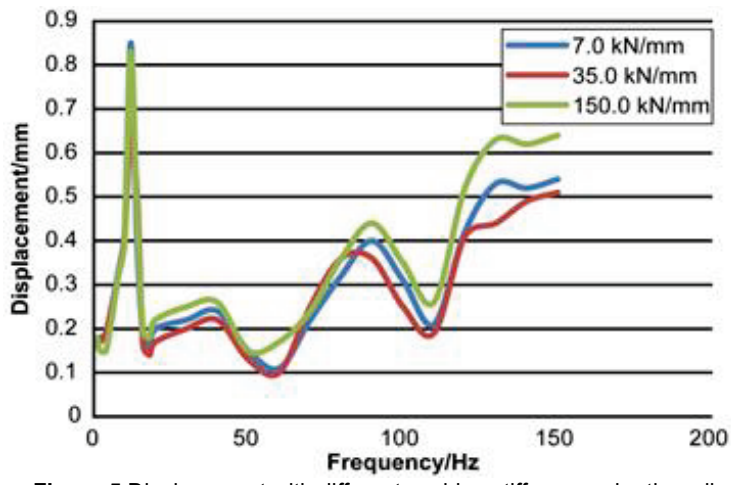

Figure 5 Displacement with different cushion stiffness under the rail

From the vibration characteristics of the system considering the frequency range, the displacement of the rail and the bearing block peaked at approximately $12 \mathrm{~Hz}$; many resonances appear, but the amplitudes are relatively small. With the increase of stiffness, the high frequency vibration of the rail and the bearing block provides a larger degree of attenuation. The same rule is not true for the bridge and the frame structure, except at approximately 12 and $24 \mathrm{~Hz}$, as the maximum displacement is different. Among the rest of the frequency band, the change of the rail pad stiffness has a negligible influence on the displacement.

Increasing the stiffness of the rail pad reduces the displacement of each part, but the force to the bottom of the framework is correspondingly increased. Therefore, the stiffness of the rail pad should be within a reasonable range. The conclusion is consistent with that in literature [18].

\subsubsection{Effect of the Bearing Block Mass}

By keeping the other parameters constant and changing the mass of the bearing block, its influence on each part of the structure can be analyzed. The results of this analysis are given in Fig. 6. As the mass of the bearing block increases, the maximum displacement of the rail, bearing block, and bridge decreases, but the maximum displacement of the frame structure increases. Neither of 
the amplitude changes are large, but the force to the bottom of the frame in the high frequency phase is significantly reduced: for bearing block masses of $100 \mathrm{~kg}$ and $130 \mathrm{~kg}$, the amplitudes decrease by $145 \mathrm{kN}$ and $172 \mathrm{kN}$, respectively. Therefore, the maximum displacement amplitude of the frame structure is not very big, and increasing the mass of the bearing block has a positive effect on improving the vibration characteristics of the system.

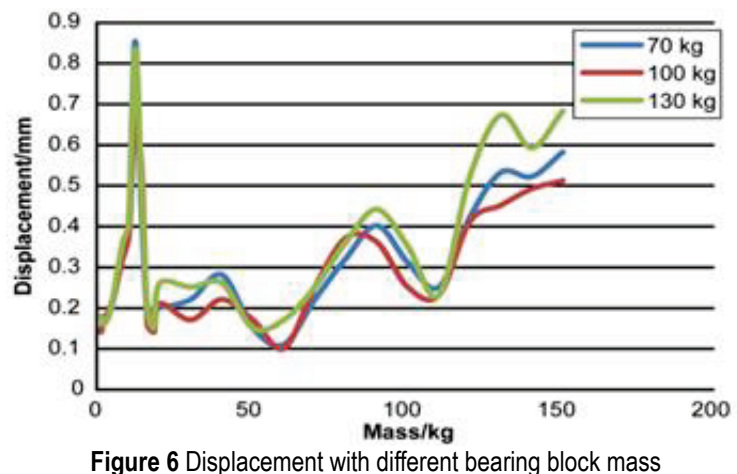

\subsubsection{Effect of Cushion Stiffness under the Bearing Block}

By keeping the other parameters constant and changing the stiffness of the cushion under the bearing block, its influence on each part of the structure can be analyzed. The results of this analysis are given in Fig. 7.

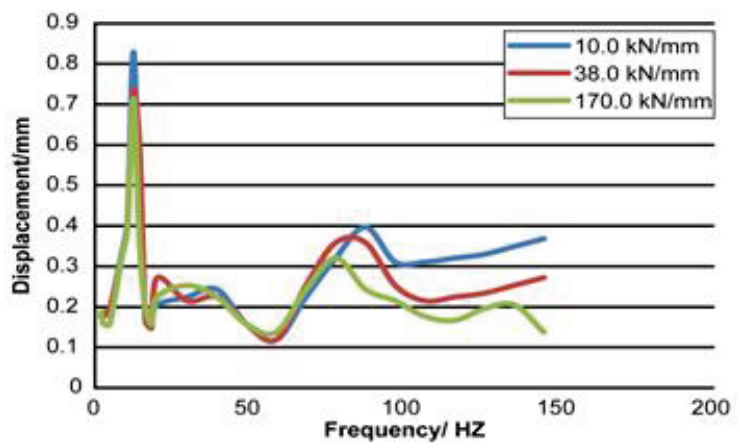

Figure 7 Displacement with different cushion stiffness under the bearing block

As the cushion stiffness increases, the maximum displacement of each part of the system decreases, and the force transmitted to the bottom of the frame also decreases. When the stiffness changes from $14.2107 \mathrm{~N} / \mathrm{m}$ to 3.8107 $\mathrm{N} / \mathrm{m}$, the force is reduced by $114 \mathrm{kN}$. For the rail and bearing block, the increasing cushion stiffness not only reduces the displacement amplitude of the first resonance, but also reduces the displacement amplitude of each high frequency resonance beyond $12 \mathrm{~Hz}$. As a result, increasing the cushion stiffness improves the vibration characteristics of the entire system.

\subsubsection{Effect of Bridge Bearing Vertical Stiffness}

By keeping the other parameters constant and changing the vertical stiffness of the bridge bearing, its influence on each part of the structure can be analyzed. The results of this analysis are given in Fig. 8.

Overall, as the vertical stiffness of the bridge bearing increases, the displacement amplitudes of the rail, bearing block, bridges, and the second layer of the framework all increase. Under these conditions, the reaction force of the bottom framework also increases, but the displacement amplitude of the third layer of the framework decreases. When the stiffness value is $4.0 \times 108 \mathrm{~N} / \mathrm{m}$, the maximum displacement of the third layer of the framework is 0.771 $\mathrm{mm}$, which is greater than that of the various parts of the upper structure. The results show that the resonance of the frame structure is much more serious. Integrated with the other parameters, the vertical stiffness values of the bridge bearing should be optimized for the vibration characteristics of the entire system.

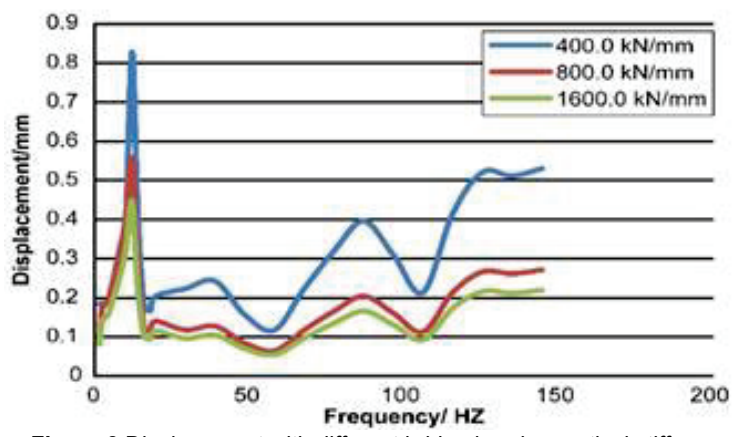

Figure 8 Displacement with different bridge bearing vertical stiffness

Table 1 Amplitude of vibration characteristics of different parts of frame structures with change of elastic modulus

\begin{tabular}{|l|c|c|c|}
\hline Elastic modulus $\left(\mathrm{N} / \mathrm{m}^{2}\right)$ & $2.3 \times 10^{10}$ & $2.9 \times 10^{10}$ & $3.3 \times 10^{10}$ \\
\hline Rail displacement $(\mathrm{mm})$ & 2.401 & 2.372 & 2.353 \\
\hline Rail acceleration $\left(\mathrm{m} / \mathrm{s}^{2}\right)$ & 99.53 & 99.53 & 99.53 \\
\hline Bearing block displacement $(\mathrm{mm})$ & 1.587 & 1.575 & 1.569 \\
\hline Bearing block acceleration $\left(\mathrm{m} / \mathrm{s}^{2}\right)$ & 70.13 & 70.13 & 70.13 \\
\hline Bridge displacement $(\mathrm{mm})$ & 0.921 & 0.892 & 0.871 \\
\hline Bridge acceleration $\left(\mathrm{m} / \mathrm{s}^{2}\right)$ & 4.71 & 4.71 & 4.71 \\
\hline Bottom reaction of frame $(\mathrm{kN})$ & 148.103 & 147.924 & 147.535 \\
\hline
\end{tabular}

\subsubsection{Influence of Elasticity Modulus of Frame Structure}

By keeping other parameters constant and changing the elasticity modulus of the frame structure, its influence on each part of the structure can be analyzed.

Varying the elastic modulus of the frame structure makes the vibration characteristics of each part of the system more complex. As the elastic modulus increases, the maximum displacements of the rail, bearing block, and bridge decrease, whereas the maximum displacements of the framework layer increase. The maximum displacement of the three-layer framework initially increases before decreasing, yet the reaction force at the bottom of the frame exhibits the opposite trend. Furthermore, as the elastic modulus varies, the resonance frequencies of the system parts change and additional resonances appear.

Therefore, the elastic modulus of the frame structure has a unique influence on each part of the system. When designing a structure, the dynamic response of each piece should undergo comprehensive consideration, choosing from reasonable grading materials, to make sure that the overall vibration control is tolerable.

\section{VERIFICATION AND DISCUSSION}

Comparing the results of the model calculation with the monitoring data of a three-storey viaduct station on the Pearl Line of Shanghai Rail Transit, the model parameters 
are as follows: $60 \mathrm{~kg} / \mathrm{m}$ rail is selected, mass of bearing block is $100 \mathrm{~kg}$, cushion stiffness under the rail is 35 $\mathrm{kN} / \mathrm{mm}$, cushion stiffness under the bearing block is 38 $\mathrm{kN} / \mathrm{mm}$, bridge bearing vertical stiffness is $800 \mathrm{kN} / \mathrm{mm}$, elastic modulus of frame beam is $2.9 \times 10^{10} \mathrm{~N} / \mathrm{m}^{2}$. As shown in Tab. 2, the difference between the two is not significant, which proves the credibility of the conclusions drawn in this paper.

Table 2 Comparison of the results of the model calculation with the monitoring

\begin{tabular}{|c|c|c|c|}
\hline & 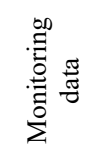 & 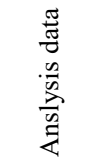 & . \\
\hline Rail displacement (mm) & 2.567 & 2.372 & 8.22 \\
\hline Rail acceleration $\left(\mathrm{m} / \mathrm{s}^{2}\right)$ & 103.23 & 99.53 & 3.72 \\
\hline Bearing block displacement $(\mathrm{mm})$ & 1.363 & 1.575 & 11.93 \\
\hline Bearing block acceleration $\left(\mathrm{m} / \mathrm{s}^{2}\right)$ & 64.83 & 70.13 & 7.56 \\
\hline Bridge displacement $(\mathrm{mm})$ & 0.995 & 0.892 & 11.55 \\
\hline Bridge acceleration $\left(\mathrm{m} / \mathrm{s}^{2}\right)$ & 4.91 & 4.71 & 4.25 \\
\hline Bottom reaction of frame $(\mathrm{kN})$ & 129.342 & 147.924 & 12.56 \\
\hline
\end{tabular}

According to Tab. 1, for the displacement of rail and support block, the measured value is larger than the theoretical value, while the theoretical value is larger than the measured value for acceleration and support reaction. The main reason is that the emphasis of finite element calculation is on track structure, the foundation under block is rigid, and the factors such as elastic-plastic deformation and beam vibration caused by wheel-rail impact are not taken into account. However, the reliability of theoretical analysis is verified by errors less than $15 \%$.

\section{CONCLUSION}

To support the increasing application of elevated stations in urban rail transit construction, with the help of related literature and the ANSYS software, a threedimensional dynamic finite element model of an elevated station-track structure system is established. The majority of the station body structure is built from space frame structures, and the track structure is built with elastic supporting block-type ground tracks. The different parts of the system components and connections are considered in this article, and modal analysis is applied to the model. The first natural frequency and the corresponding vibration mode of the system are obtained. Based on the modal analysis, the vibration characteristics under harmonic load and moving train load are analyzed. In the harmonic analysis, the main consideration is the effect of the vibration characteristics of the system from the change of the rail pad stiffness, the mass of the bearing block, the cushion layer stiffness, the vertical stiffness, the bearing stiffness, and the framework structure elastic modulus

Through the modal analysis of the elevated stationtrack structure system, it can be seen that the first six frequency and vibration modes of the system are mostly caused by the structure frame; furthermore, the maximum displacements of the mode shapes also appeared in the frame structure. From the vibration mode shape, the first order vibration mode of the structure is longitudinal bending deformation, which shows that the longitudinal stiffness is smaller than its lateral stiffness. Therefore, more attention should be paid to the longitudinal stability of the structure in practical engineering situations.

The reasonable matching between the rail pad stiffness and the cushion stiffness has great influence on the system vibration characteristics. Through the comparison and analysis of the stiffness of the rail pad and cushion under the rail and block, respectively, the vibration characteristics of the control system are much better when the stiffness of the rail pad is smaller than that of the cushion under the block.

\section{Acknowledgements}

This work was supported by the Sichuan Science and Technology Program (Grant No. 2019JDJQ0018) and supported by the Fundamental Research Funds for the Central Universities (Grant No. 2682018GJ02).

\section{REFERENCES}

[1] Kaynia, A. M., Madshus, C., \& Zackrisson, P. (2000). Ground vibration from high-speed trains: prediction and countermeasure. Journal of Geotechnical and Geoenvironmental Engineering, 126(6), 531-537. https://doi.org/10.1061/(ASCE)1090-0241(2000)126:6(531)

[2] Xia, H., Zhang, N., \& Cao, Y. M. (2005). Experimental study of train-induced vibrations of environments and buildings. Journal of Sound and Vibration, 280, 1017-1029. https://doi.org/10.1016/j.jsv.2004.01.006

[3] Ju, S. H., Lin, H. T., \& Chen, T. K. (2007). Studying characteristics of train-induced ground vibrations adjacent to an elevated railway by field experiments. Journal of Geotechnical and Geoenvironmental Engineering, 133(10), 1302-1307. https://doi.org/10.1061/(ASCE)1090-0241(2007)133:10(1302)

[4] Tanabe, M., Matsumoto, N., Wakui, H., Sogabe, M., Okuda, H., \& Tanabe, Y. (2008). A simple and efficient numerical method for dynamic interaction analysis of a high-speed train and railway structure during an earthquake. Journal of Computational and Nonlinear Dynamics, 3(4), 1-8. https://doi.org/10.1115/1.2960482

[5] Yang, N., Guo, T., \& Sun, G. Z. (2013). Train-induced vibration on elevated railway station. Journal of Central South University, 20(12), 3745-3753. https://doi.org/10.1007/s11771-013-1903-2

[6] Ren, C., Xu, Z., Xiao, C., \& Li, L. (2014). Seismic performance analysis of single-column elevated station considering pile-soil interaction. Journal of Building Structures, 35(5), 25-32.

[7] Tanabe, M., Sogabe, M., Wakui, H., Matsumoto, H. N., \& Tanabe, Y. (2015). Exact time integration for dynamic interaction of high-speed train and railway structure including derailment during an earthquake. Journal of Computational and Nonlinear Dynamics, 11(3), 1-12. https://doi.org/10.1115/1.4030829

[8] Prusty, B. G. \& Satsangi, S. K. (2001). Finite element transient dynamic analysis of laminated stiffened shells. Journal of Sound and Vibration, 248(2), 215-233. https://doi.org/10.1006/jsvi.2001.3678

[9] Taktak, M., Dammak, F., Abid, S., \& Haddar, M. (2008). A finite element for dynamic analysis of a cylindrical isotropic helical spring. Journal of Mechanics of Materials and Structures, 3(4), 641-658. https://doi.org/10.2140/jomms.2008.3.641

[10] Zhu, D., Chen, H., Kong, X., \& Zhang, W. (2014). A hybrid finite element-energy finite element method for midfrequency vibrations of built-up structures under multi- 
distributed loadings. Journal of Sound and Vibration, 333(22), 5723-5745. https://doi.org/10.1016/j.jsv.2014.06.024

[11] Chan, H. C., Cai, C. W., \& Cheung, Y. K. (1993). Convergence studies of dynamic analysis by using the finite element method with lumped mass matrix. Journal of Sound and Vibration, 165(2), 193-207. https://doi.org/10.1006/jsvi.1993.1253

[12] Lou, P., Dai, G. L., \& Zeng, Q. Y. (2007). Dynamic analysis of a Timoshenko beam subjected to moving concentrated forces using the finite element method. Shock Vib, 14(6), 459-468. https://doi.org/10.1155/2007/460206

[13] Jorge, P. C., da Costa, A. P., \& Simões. F. M. F. (2015). Finite element dynamic analysis of finite beams on a bilinear foundation under a moving load. Journal of Sound and Vibration, 346, 328-344. https://doi.org/10.1016/j.jsv.2014.12.044

[14] Al-Khafaji, A. A. M. \& Mat Darus, I. Z. (2014). Finite element method to dynamic modelling of an underwater flexible single-link manipulator. Journal of Vibroengineering, 16(7), 3620-3636.

[15] Wang, D., Xie, M., \& Li, Y. (2015). High-frequency dynamic analysis of plates in thermal environments based on energy finite element method. Shock Vib, 2015(2), 1-14. https://doi.org/10.1155/2015/157208

[16] Wang, Y., Wang, S., Zhao, Y., Li, X., Guo, P., \& Liu, Y. (2019). Analysis of fracturing characteristics of unconfined rock plate under edge on impact loading. European Journal of Environmental and Civil Engineering. https://doi.org/10.1080/19648189.2018.1509021

[17] Yuan, B., Sun, M., Wang, Y., Zhai, L., Luo, Q., \& Zhang, X. (2018). A full 3D displacement measuring system for 3D displacement field of soil around a laterally loaded pile in transparent soil. International Journal of Geomechanics. https://doi.org/10.1061/(ASCE)GM.1943-5622.0001409.

[18] Zhu, J. Y. \& Geng, C. Z. (2005). Comparison Investigation on Dynamic Responses of Elevated Low Vibration Track Under Wheel Load Drop. Journal of Tongji University, 33(12), 1621-1625.

\section{Contact information:}

\section{Kai CUI}

Coresponding author

Key Laboratory of High-speed Railway Engineering of the Ministry of Education, Southwest Jiaotong University, Chengdu, Sichuan, 610031, P. R. China

E-mail: cuikai@swjtu.cn

\section{Lei SU}

School of Civil Engineering, Southwest Jiaotong University, Chengdu, Sichuan, 610031, P. R. China 